\title{
Correction to: Neural networks catching up with finite differences in solving partial differential equations in higher dimension
}

\section{Vsevolod I. Avrutskiy ${ }^{1}$}

Published online: 10 April 2020

(C) Springer-Verlag London Ltd., part of Springer Nature 2020

\section{Correction to: Neural Computing and Applications} https://doi.org/10.1007/s00521-020-04743-8

Unfortunately, Figs. 1, 2 and 3 and their respective captions have been placed incorrectly in the online publication of the article.

The correct Figs. 1, 2 and 3 and their captions are given below.

The original article has been updated accordingly.

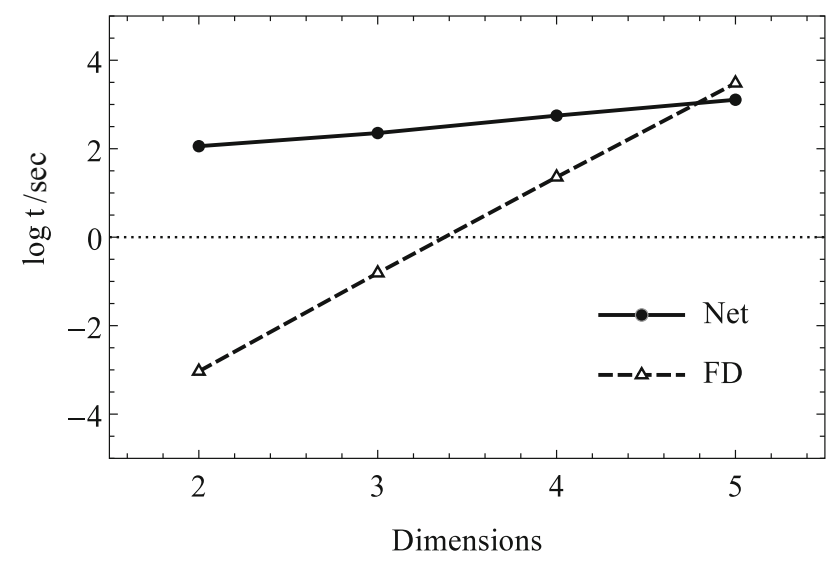

Fig. 1 Base 10 logarithms of solving time in seconds for neural networks and projected running time for the second-order finite difference scheme

The original article can be found online at https:// doi.org/10.1007/s00521-020-04743-8.

\section{Vsevolod I. Avrutskiy}

avrutsky@phystech.edu

1 Department of Aeromechanics and Flight Engineering, Moscow Institute of Physics and Technology, Institutsky Lane 9, Dolgoprudny, Moscow Region, Russia 141700

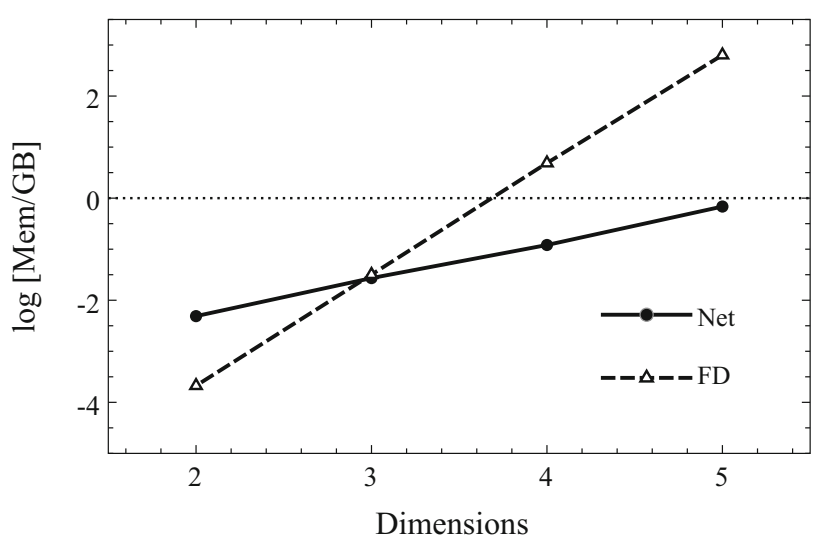

Fig. 2 Base 10 logarithms of memory in GB for neural network method and the second-order finite difference scheme

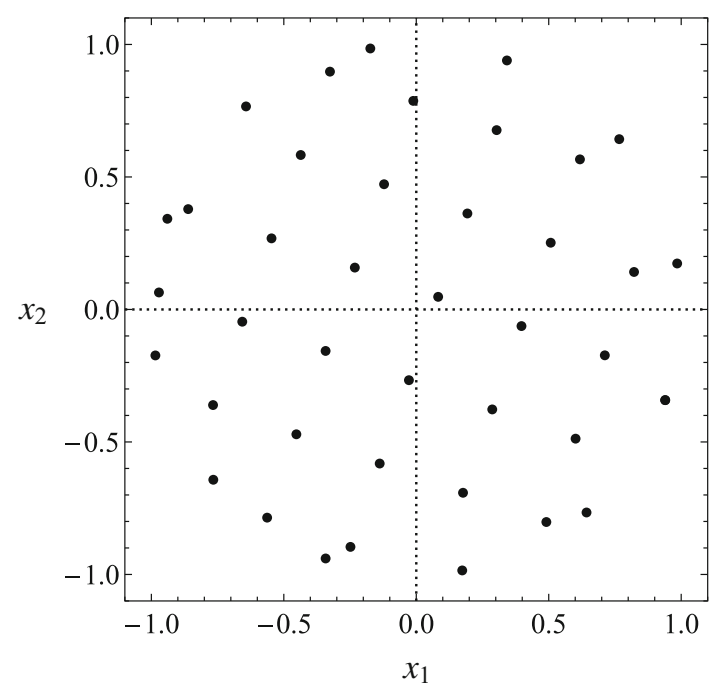

Fig. $32 \mathrm{D}$ grid with spacing $\lambda=1 / 3$ for linear Poisson equation. The same $\lambda$ is used in higher dimensions

Publisher's Note Springer Nature remains neutral with regard to jurisdictional claims in published maps and institutional affiliations. 